\section{$\underset{\text { \&ommes migrations }}{\text { homm }}$}

\section{Hommes \& migrations}

Revue française de référence sur les dynamiques

migratoires

$1304 \mid 2013$

Frontières

\title{
Des frontières de la migration aux représentations de l'Europe
}

\section{Anaïk Pian}

\section{(2) OpenEdition}

\section{Journals}

Édition électronique

URL : http://journals.openedition.org/hommesmigrations/2661

DOI : 10.4000/hommesmigrations.2661

ISSN : 2262-3353

Éditeur

Musée national de l'histoire de l'immigration

Édition imprimée

Date de publication : 1 octobre 2013

Pagination : 11-17

ISBN : 978-2-919040-24-7

ISSN : $1142-852 X$

Référence électronique

Anaïk Pian, "Des frontières de la migration aux représentations de l'Europe », Hommes \& migrations [En ligne], 1304 | 2013, mis en ligne le 01 janvier 2017, consulté le 19 avril 2019. URL : http:// journals.openedition.org/hommesmigrations/2661; DOI : 10.4000/hommesmigrations.2661 
Près de la forêt de Bel Younes, frontière Maroc-Espagne

(Ceuta), côté marocain, le 21 octobre 2007.

() Anaïs Pachabézian-Babel Photo 


\section{DES FRONTIÈRES \\ DE LA MIGRATION AUX REPRÉSENTATIONS DE L'EUROPE}

par ANAÏK PIAN, sociologue, maître de conférences au laboratoire Cultures et sociétés en Europe, université de Strasbourg.

La proximité géographique entre le Maroc et l'Europe conduit de nombreux migrants subsahariens à tenter le passage de cette frontière clandestinement. Cependant, l'ailleurs rêvé, si proche, n'en finit pas de s'éloigner à mesure des revers de leur aventure. Les frontières de l'Europe se transforment en impasse, comme I'ont montré en 2005 les tentatives désespérées des migrants africains pour franchir en nombre les barrières de Ceuta et Melilla, les deux enclaves espagnoles sur le continent africain. Qu'en est-il, dans ce contexte, des représentations de l'Europe?

\section{Entre frontières politiques et géographiques}

Depuis plus d'une dizaine d'années, l'externalisation du contrôle des frontières est une ligne directrice des politiques d'immigration européennes. Instituant une "forme de police à distance et à distemps ${ }^{1 "}$, ce mode de gouvernement international des migrations ${ }^{2}$ implique de repousser toujours plus en amont la lutte contre l'immigration clandestine et le filtrage des migrants autorisés à accéder à l'espace Schengen. Il participe, par effet domino, à la création de nouveaux espaces dits "de transit", d'espace-temps de l'entre-deux ${ }^{3}$ ou "d'encampement", invitant à une réflexion sur

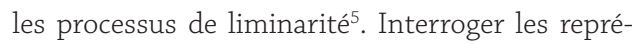
sentations de l'Europe dans ce contexte conduit à accorder une importance aux frontières. Celles-ci font partie intégrante des représentations de l'Europe des migrants, qu'elles soient appréhendées d'un point de vue pratique, symbolique, géographique ou politique. Procédant "d'espaces cognitifs de références, participant de la production de catégo- 
ries, de modes de légitimation ou d'exclusion ${ }^{6 "}$, elles impliquent aussi une position ou un rapport au monde, à soi, et aux autres. "Où l'Europe commencet-elle? se demandent les émigrés qui cherchent à devenir immigrés. Aux frontières Schengen, à l'aéroport de Bamako, ou dans la 'jungle' de Calais, dont Nicolas Sarkozy avait assuré avoir résolu le problème en fermant Sangatte?" Cette citation, empruntée à JeanFrançois Bayart ${ }^{7}$, montre

Depuis le début des années 2000, le territoire marocain est devenu une voie de passage privilégiée vers l'Europe pour quelques milliers de Subsahariens n'ayant pas les moyens légaux d'immigrer. la labilité des représentations des frontières selon le point de vue.

C'est pourquoi celles-ci méritent d'être appréhendées de manière socialement située, le social étant luimême étroitement intriqué à des dimensions spatiales et temporelles. À partir de la situation des migrants subsahariens cherchant à rejoindre clandestinement les pays européens depuis le territoire marocain, l'article interroge deux dimensions : comment les pratiques de la migration contribuent-elles à (re)façonner les représentations de l'Europe et de ses frontières? De quelle manière l'expérience des frontières contribue-t-elle à alimenter, voire à modifier, certaines représentations de l'Europe? Une attention particulière sera portée à la façon dont la confrontation à la Guardia Civil, au niveau des enclaves espagnoles de Ceuta et Melilla, alimente chez les migrants de nouvelles représentations de l'Europe à la fois en tant qu'espace politique et sur le plan des relations interethniques. La présente contribution repose sur deux enquêtes ethnographiques : la première a été réalisée entre 2003 et 2006 dans le cadre d'un doctorat de sociologie ; la seconde en 2009 dans le cadre du projet européen Eurobroadmap ${ }^{8}$ sur les représentations dans le monde. Au total, plus d'une centaine de migrants originaires d'Afrique centrale et de l'Ouest ont été rencontrés, principalement dans les villes de Rabat, Casablanca et Fès. Auprès d'une partie d'entre eux, l'établissement d'une relation de longue durée a permis un suivi de leur trajectoire.

\section{La politique des visas : le vocabulaire de la chance et de l'arbitraire}

Depuis le début des années 2000, le territoire marocain est devenu une voie de passage privilégiée vers l'Europe pour quelques milliers de Subsahariens n'ayant pas les moyens légaux d'immigrer. Avant de se rendre au Maroc, nombre d'entre eux ont essayé d'obtenir, depuis leur pays d'origine (Congo, Cameroun, Sénégal, Mali, Guinée...), un visa pour l'Europe, mais se sont heurtés à des procédures administratives perçues comme particulièrement opaques. En témoignent les expressions "gagner des visas", "avoir la chance d'aller en Europe" ou encore "être né du mauvais côté" qui reviennent fréquemment à l'évocation de ces expériences; et ce, quels que soient le type de visa demandé (étudiant, court séjour, etc.), les consulats auprès desquels ont été effectuées les démarches (France, Italie, Portugal...) ou encore le profil sociologique des demandeurs déboutés.

La référence au destin et/ou à l'arbitraire prend le pas sur le cadre rationnel et légal, y compris lorsque les intéressés ont quelques connaissances des politiques d'immigration européennes.

Si les refus en matière de visas alimentent un profond sentiment d'injustice, les consulats et gouvernements européens ne sont pas les seuls incriminés. L'apparatchik des gouvernements africains est aussi mis en cause, car pour "gagner le visa", il faut "avoir le bras long" et des relations de cooptation avec des intermédiaires se chargeant de démarcher. Suite à un premier refus de visa, voire avant toute première tentative, certains migrants ont fait appel à des "affaires men" ou "coxers" pour tenter d’obtenir le précieux docu- 
ment, mais le prix, souvent fort cher, ne protège pas contre les arnaques et ne garantit pas le succès de l'entreprise.

\section{Les routes de l'aventure}

Face à l'impossibilité d'obtenir un visa, les "routes de l'aventure" apparaissent pour de nombreux candidats à la migration comme la seule solution pour atteindre l'Europe. Leurs représentations de l'Europe par le rapport aux frontières font intervenir une double dimension, géographique et politique, qui évolue au cours de l'expérience migratoire.

Le choix du Maroc pour rejoindre l'Europe s'appuie souvent, même s'il se combine à d'autres dimensions (expériences migratoires antérieures, contacts et réseaux, etc.), sur la proximité géographique entre le Maroc et l'Espagne : les quelques kilomètres (14 km) séparant les côtes marocaines de la péninsule espagnole au niveau du détroit de Gibraltar, les enclaves de Ceuta et Melilla et même les îles Canaries figurent comme des symboles de cette apparente accessibilité/ proximité. Orientant les pratiques spatiales de la migration, ces représentations géographiques revêtent une fonction prescriptive.

Pour autant, avant d'arriver au Maroc, les intéressés n'ont pas toujours une connaissance précise des différentes tactiques de passage, ou des lieux d'où elles s'organisent, ni des créations d'institutionnalisation ad hoc ${ }^{9}$ sur lesquelles elles s'appuient. Et ce, quand bien même le voyage a fait l'objet d'une préparation en amont. Lorsque le virtuel devient réel, les étapes se précisent, qu'il s'agisse des villes à traverser, des transports à emprunter, des codes à respecter ou encore des dangers à éviter... À cela s'ajoute l'affirmation récurrente selon laquelle pour "croire" ce qui se dit sur ces routes migratoires, il faut aller voir soi-même. Si la localisation des portes d'entrée de l'Europe s'affine au cours de l'expérience migratoire, de nombreux migrants prennent conscience que la distance géographique n'est pas en soi l'obstacle à franchir : celui-ci se situe plutôt du côté des dispositifs de contrôle dans le contexte d'externalisation de la lutte contre l'immigration clandestine : "Avantj'envisageais ça plus facilement, je me disais maintenant que je suis au Maroc, il suffit que j'aie mon argent et je passe. Je ne savais pas que je buterais sur une frontière quill n'est pas possible de passer (...). Maintenant, je me suis rendu compte que c'est la politique de

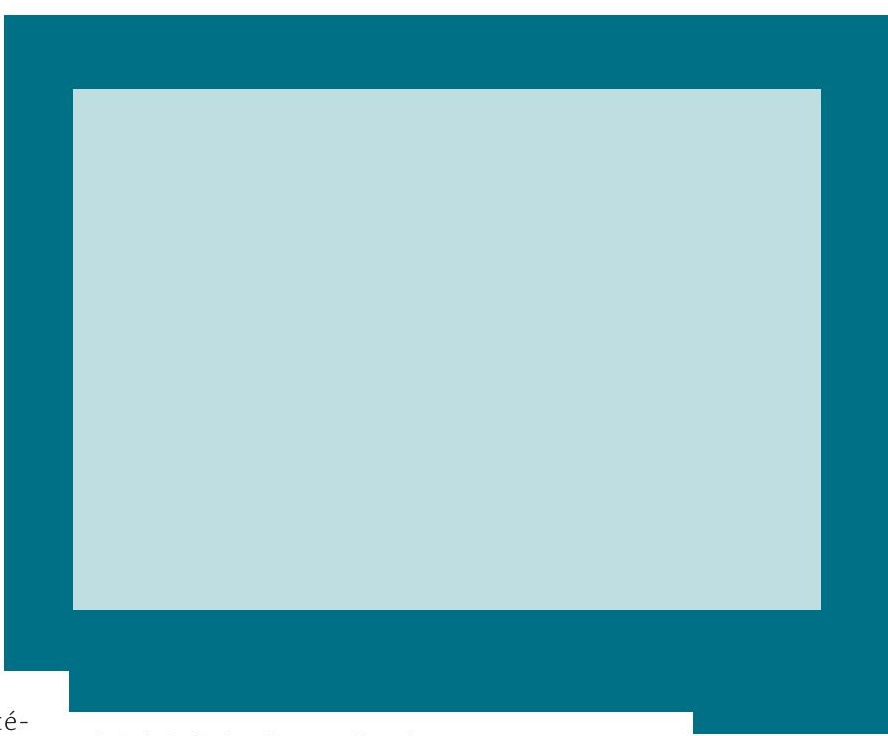

Près de la forêt de Bel Younes, frontière Maroc-Espagne (Ceuta), côté marocain, le 21 octobre 2007

(c) Anaís Pachabézian-Babel Photo

l'Europe. J'ai compris que tout ce que je suis en train de vivre, c'est parce que c'est une politique européenne, parce qu'on ne veut pas de nous, parce qu'on veut une certaine politique économique. J'ai pu comprendre que tout ce que je suis en train de vivre, c'est programmé, c'est orienté... Je ne savais pas ça avant. J'étais dedans, mais je ne savais pas" (Congolais, la trentaine, "coincé" au Maroc depuis plusieurs années). Pour plusieurs migrants, en effet, l'expérience marocaine s'accompagne d'une socialisation poli- 
tique et militante (avec parfois, mais pas nécessairement, des expériences antérieures dans le pays d'origine). Au cours de la migration, celle-ci a été progressivement acquise au contact d'une forte mobilisation associative et de la société civile qui, à partir du milieu des années 2000, se développe de façon exponentielle au À partir de la fin $\quad M_{a r o c}{ }^{10}$, en réaction au traides années 1990, face à tementdela question migral'ampleur prise par les arrivées continues de nouveaux migrants et les pressions politico-économiques ainsi générées, le gouvernement espagnol renforce

la militarisation des enclaves de Ceuta et Melilla. toire par les gouvernements marocain et européens. Ces mobilisations se traduisentd'uncôtéparl'action (voire la création) de nombreuses associations nationales, internationales et d'ONG défendant la cause des migrants subsahariens, que ce soit à travers un filtre à dominance humanitaire, caritatif, juridique et/ou de plaidoyer.

De l'autre, elles s'expriment par la multiplication des associations de migrants revendiquant une visibilité dans l'espace public marocain et même international et le respect des droits fondamentaux dans un contexte de répression accrue à leur encontre.

En 2005, une première formation sur le droit d'asile organisée par la Cimade et l'Afvic (Association des amis et familles des victimes de l'immigration clandestine $^{11}$ ) avec le soutien financier du Haut Commissariat aux réfugiés (HCR) se tient ainsi à Bouznika, à laquelle participent plusieurs migrants dont quelques-uns deviendront des leaders associatifs. Ce passage à la politique, en tant que "prises de

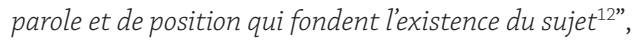
participe de la redéfinition des représentations de l'Europe et de ses frontières par ceux qui n'ont pas l'autorisation de les franchir. Ces prises de parole sont aussi à replacer au sein d'événements catalyseurs symbolisant le processus de frontiérisation à l'entrée de l'espace Schengen : les violences à Ceuta et à Melilla de l'automne 2005.

\section{La confrontation à un espace frontière : les enclaves espagnoles de Ceuta et Melilla}

Jusqu'au début des années 2000, les enclaves espagnoles de Ceuta et Melilla, situées au nord du Maroc et intégrées à l'espace Schengen en 1991, étaient perçues comme une porte permettant d'accéder à l'Europe sans prise de risques majeurs, c'est-à-dire sans mise en péril de sa vie. En passant par ces enclaves, il n'y avait pas de mer à traverser, seulement des grillages à escalader en déjouant la surveillance des autorités marocaines et espagnoles.

Des migrants ayant réussi à pénétrer dans les enclaves espagnoles à la fin des années 1990 et ayant séjourné dans divers pays européens (Espagne, Portugal, Allemagne, etc.) avant d'être rapatriés dans leur pays d'origine (puis de reprendre la route par le Maroc) gardent de cette époque une image de la Guardia Civil beaucoup moins violente que celle d'aujourd'hui. D'autres, "bloqués" sur le territoire marocain depuis plusieurs années, insistent sur le fait qu'au cours des années 2000-2003, le plus difficile n'était pas tant de réussir à franchir les grillages protégeant les enclaves, que de parvenir aux "campos", camps où ils devaient séjourner le temps que leur éventuelle requête d'asile soit traitée, que leur nationalité soit établie par les autorités espagnoles et/ ou qu'un permis humanitaire leur soit éventuellement attribué.

"À l'époque c'est la Guardia qui avait le pouvoir sur tout à Melilla. Tu allais au commissariat, on te donnait un récépissé et avec ça tu pouvais aller dans le camp. Mais tout ça c'était un truc... Pourquoi quand tu

10. Anaïk Pian, "Entre visibilisation et invisibilisation, les migrations subsahariennes au Maroc", in Ali Bensaad (dir.),

Le Maghreb à l'épreuve des migrations transsahariennes. Immigration sur émigration, Paris, Karthala, 2009, pp. 65-88; Laura Feliu Martínez, "Les migrations en transit au Maroc. Attitudes et comportement de la société civile face au phénomène", in L'Année du Maghreb, vol. V, 2009, pp. 343-362. 11. Cette association marocaine se préoccupait à son origine de la question de l'immigration clandestine des Marocains. 12. Michel Agier, Gérer les indésirables. Des camps de réfugiés au gouvernement humanitaire, Paris, Flammarion, 2008. 
rentres tu ne vas pas directement au camp? C'était un moyen des politiques pour que la Guardia nous arrête. Moi j’ai eu le reçu au commissariat mais, en sortant, la Guardia m'a arrêté, ils m'ont tapé. Alors qu'à l'époque, on disait que le plus dur c'était de passer le grillage (...). Moi, je peux dire que le plus dur cétait de réussir à avoir le récépissé et à aller au camp" (Sénégalais, 37 ans). À travers ce discours, les modalités du contrôle des frontières intérieures apparaissent encore plus arbitraires que le contrôle des frontières extérieures.

À partir de la fin des années 1990, face à l'ampleur prise par les arrivées continues de nouveaux migrants et les pressions politico-économiques ainsi générées, le gouvernement espagnol renforce la militarisation des enclaves de Ceuta et Melilla. Tandis que la répression s'accroît de part et d'autre des territoires marocain et espagnol, les représentations des frontières de l'Europe commencent à changer pour le regard de nombreux migrants. Dans la nuit du 28 au 29 septembre 2005 puis, quelques jours plus tard, dans celle du 5 au 6 octobre, plusieurs centaines de ressortissants subsahariens tentent de pénétrer dans les enclaves espagnoles. Les autorités espagnoles et marocaines réagissent par un revirement sécuritaire sans précédent : elles font usage d'armes à feu et tous les migrants interceptés sont refoulés vers le Maroc après avoir été maltraités par la Guardia Civil (et sans qu'il leur soit permis de déposer une demande d'asile).

Des expulsions inédites ont lieu au sud de la frontière algéro-marocaine et au niveau de la frontière mauritanienne. Pour les migrants concernés, cette confrontation directe à la Guardia Civil alimente trois types de représentations où s'imbriquent les rapports aux frontières et à leur gouvernementalité, au territoire et à ceux qui l'habitent. En effet, si les frontières produisent des étrangers sur le plan juridique, elles fabriquent aussi des étrangers anthropologiques ${ }^{13}$ sur le plan des relations interethniques.

\section{Monopole et limites de la violence légitime}

Pour certains, la violence de la Guardia Civil espagnole à l'automne 2005 est perçue comme une violence d'exception dans une zone singulière liée à l'espace frontière. Les pratiques de la Guardia Civil ne sont pas considérées comme représentatives de l'attitude des Espagnols dans leur ensemble et encore moins des Européens en général. Elles sont davantage attribuées à des fonctionnaires de l'institution militaire et policière chargés d'empêcher les entrées clandestines sur le territoire espagnol, soit à des "gens qui font leur travail". Des migrants reconnaissent la légitimité de la défense de leurs frontières par les Européens - ici, les Espagnols - au nom du principe de souveraineté tel que l'entend Benedict Anderson" ${ }^{14}$ : "La Guardia, des fois, lorsquills tont attrapé, ils peuvent mettre la musique dans la voiture, ils te disent de danser. Pour les faire rire... ils te disent, morino, morino... Mais si vous tentez en masse [le passage du grillage], ils La violence aux frontières dans le périmètre frontalier de Ceuta et Melilla a pu impulser une rupture brutale avec les représentations antérieures de l'Europe. peuvent tirer des gaz lacrymogènes pour vous empêcher, pour vous évanouir, pour quills puissent vous prendre et vous faire sortir [du côté marocain]. Moi... javais en tête que cétait des gens de la sécurité. Ils sont là pour faire leur travail, qui consiste à nous empêcher de rentrer. Ils sont dans l'exercice de leurs fonctions. Donc moi ça ne pouvait pas me donner l'image de l'Espagne, que si je rentre, soi-disant c'est comme ça en Espagne. Je serai traité comme un homme. Je sais que ceux (les Espagnols) qui se trouvent dedans, et ceux qui sont à la sécurité, n’ont pas les mêmes stratégies, les mêmes visions", (Camerounais, la trentaine). D'autres reprennent les discours communs, assez répandus en Europe, selon lesquels les pays occidentaux ne peuvent accueillir tous les Africains ni 
"toute la misère du monde" à l'instar de la célèbre formule du politicien français Michel Rocard, même s'ils ne savent pas exactement à qui l'attribuer. Se retrouve à travers ces propos un "modèle de perception des mobilités et de la présence détrangers" faisant écho au "risque d'invasion"15. Néanmoins, même si certains migrants reconnaissent la légitimité du contrôle des frontières, la violence de la Guardia Civil lors des événements de Ceuta et Melilla en 2005 marque pour eux un débordement inédit, le dépassement d’une limite légitime.

\section{Illégitimité et rupture}

La violence aux frontières dans le périmètre frontalier de Ceuta et Melilla a pu impulser une rupture brutale avec les représentations antérieures de l'Europe. Les valeurs fondamentales que les migrants associaient aux pays européens dans leur ensemble (démocratie, liberté, respect, etc.) et, en filigrane, à la catégorie générique de "toubab" sont définitivement, disent-ils, remises en cause. Dès lors, c'est la frontière morale marquant la distinction entre des "eux" (Européens) et des "nous" (Africains) qui se brouille, car, à leur grande désillusion, les premiers ne valent pas mieux que les seconds en matière de respect des droits humains. "Lorsqu'on a fait l'attaque forcée et que j'ai vu qu'on tirait sur des gens, jétais en colère, je pouvais être gravement blessé, je pouvais mourir là-bas... Parce que je ne me contrôlais pas, je criais... C'est après, je me suis calmé... Je suis fou, qu'est-ce qui m'a pris? Je n'étais pas gravement blessé, j’ai remercié le bon Dieu... J'étais déçu vraiment de la Guardia qui tire comme ça sur les gens, sans pitié... sans états d'âme... et avec tout ça, même blessés, on vous prend... On vous sort... et les Maro- cains encore... Je les ai responsabilisés dans ça. On nous blesse là-bas, on nous frappe, on vient encore et vous nous acceptez facilement. Je me suis dit c'est la politique... Ça m'a changé l'image de l'Europe tout entière. Car ce n'était pas seulement l'Espagne. Je me suis dit tous les Blancs, ils pouvaient être là, ils pouvaient faire la même chose. Je ne croyais pas ce que je voyais là-bas. Qu'on frappe quelqu'un en Europe, qu'on tue les gens... cétait trop... je n'imaginais pas ça. Même en Afrique, je n'ai pas vu ça! Qu'on tue les gens! Et on dit que l'Europe, c'est un exemple, tout le monde veut suivre l'exemple des Européens, des Blancs... ce sont les personnes de référence, la bonté, la bonne vie..." (Guinéen, la trentaine).

\section{Des catégories ethnicisées : la Guardia Civil, des "Arabes naturalisés" ?}

Pour d'autres la violence attribuée à la Guardia Civil dans l'espace frontalier de Ceuta et Melilla se reporte, d'une part, sur l'image des Espagnols dans leur ensemble et, d'autre part, les démarque dans un même mouvement des autres Européens. L'Espagne est considérée comme l'un des pays les plus brutaux et les plus racistes d'Europe en raison de sa position de porte d'entrée de l'espace Schengen. Il reste que certains peinent à croire que des Européens, de quelque nationalité qu'ils soient, puissent traiter de manière si violente des étrangers même "sans papiers" ou "clandestins". D'aucuns se demandent même si les membres de la Guardia Civil espagnole ne sont pas des "Arabes naturalisés ${ }^{16 "}$ : dans leurs représentations, en effet, "un Européen", selon une catégorie généralisante, ne peut agir ainsi. En témoignent les propos de ce Congolais : "Et la Guardia Civil, tu as du mal à croire que c'est des vrais

15. Voir Marc Bernardot, “Invasions, subversions, contaminations. De quelques figures et lieux contemporains d'un Autre exceptionnel", in Cultures \& Conflits. Gouverner les frontières, vol. 84, 2011, http://conflits.revues.org/18237. Par ailleurs, dans les représentations de plusieurs migrants, les pays du nord de l'Europe (Suède, Finlande, Danemark) offrent des possibilités d'emploi et une intégration facilitée en raison notamment d'une présence immigrée relativement faible comparativement aux pays d'immigration " traditionnels " comme la France, mais aussi comme l'Espagne ou l'Italie qui, à partir des années 1980-1990, sont devenues des destinations de plus en plus prisées par les Ouest-Africains notamment. Pour autant, ces représentations ne signifient pas que les pays nordiques soient les destinations concrètement envisagées par les intéressés. 16. Cette représentation témoigne plus largement des relations souvent tendues que les migrants entretiennent avec les Marocains. Si dans les faits, il existe aussi de bonnes relations, celles-ci tendent systématiquement à être minimisées dans les discours. Voir Anaïk Pian, Aux nouvelles frontières de l'Europe. L'aventure incertaine des Sénégalais au Maroc, op. cit. 
Espagnols. Car tu es dans le mythe de voir les Européens comme des gens qui disent la vérité, qui sont courtois, sympas... (...) Ce qui fait que quand tu arrives, tu trouves le contraire et c'est toi-même qui te crées une justification. Tu t'es mis dans la tête que l'Européen ne peut pas arriver à ce stade. Tu leur cherches des excuses. Tu te dis que ce n'est pas eux. Que c'est des Arabes, des Marocains naturalisés mais pas des Blancs ${ }^{17}$."

Qu'ils oscillent entre l'une ou l'autre de ces représentations, les témoignages recueillis tendent à se rejoindre sur un point : la confrontation aux frontières lors des événements de Ceuta et Melilla à l'automne 2005 a entaché les représentations de l'Europe de nombreux migrants en tant que pays des droits de l'homme. Et ce, d'autant plus que la violence policière se poursuit : en témoigne dernièrement, en mars 2013 puis en septembre et novembre 2013, la forte répression dont les migrants tentant de franchir les enclaves espagnoles ont à nouveau fait les frais.

\section{Le vocabulaire du sacrifice, de la souffrance et de la double dette}

Tout en étant encore ardemment désirée, l'Europe devient en partie associée à la double expérience du sacrifice et de la souffrance (physique et morale). Celle-ci, tout d'abord, est rattachée à la mise en danger de sa propre vie et de son corps pour l'Europe. Elle renvoie ensuite à l'investissement mobilisé, que ce soit sur le plan matériel, financier ou relationnel (vivre loin des siens). Il en ressort, le temps passant, l'impression d'une vie sacrifiée ou mise entre parenthèses ou le cours d'un voyage sans fin. La mise en récit, au cours des entretiens, des souffrances endurées peut aboutir au renoncement à vouloir atteindre l'Europe à tout prix, c'est-à-dire en se mettant en danger de mort. Mais c'est bien sur le "à tout prix" qu'il convient d'insister. Oumar
(Malien) dit renoncer à vouloir partir en Europe "à l'aventure", mais il envisage de s'y rendre "de manière digne", c'est-à-dire légalement, si un jour une opportunité se présente. Thierno (Guinéen) a déposé une demande d'aide au retour auprès de l'Organisation internationale pour les migrations (OIM) à Rabat. Dans ses propos toutefois subsiste la volonté d'aller voir un jour, dans une temporalité plus lointaine, ce "qu'est" l'Europe : "Pourquoi ? Parce que j'ai souffert beaucoup pour l'Europe. Et j'ai envie de voir : est-ce que c'est pour cette Europe, que je me suis donné tant de mal à aller voir, est-ce que ça vaut la peine ?" Cette volonté de partir "voir" l'Europe dans un futur éloigné est récurrente dans les discours de ceux qui envisagent pourtant, à un moment donné, un retour au pays. Au-delà des projets migratoires initiaux (partir pour étudier, découvrir, intégrer une équipe de foot, réunir un capital, demander l'asile, etc.), cette volonté est justifiée et légitimée par le sentiment d'une double dette de l'Europe à leur égard. Si elle se réfère, dans une temporalité passée, à l'existence d'une dette coloniale, elle se réfère aussi et surtout, dans la temporalité du présent, aux sacrifices et souffrances endurés au cours de l'expérience migratoire.

Les frontières constituent un dispositif de marquage à différents niveaux, qu'il s'agisse des rapports entre États et des inégalités Nord/Sud ou des relations interethniques. À partir de l'exemple marocain, cet article apporte un éclairage sur la manière dont les frontières peuvent être interprétées et représentées par ceux qui tentent de les franchir clandestinement. In fine, le rapport aux frontières dans le contexte d'externalisation du contrôle migratoire n'invite pas tant à repenser le couple mobilité/sédentarité ${ }^{18}$ que les formes hybrides de mobilité dans l'immobilité et d'immobilité dans la mobilité : en témoignent les circulations migratoires en boucle qui se jouent dans les zones frontalières où s'opère un chassé-croisé continu entre tentatives de passage, répression et expulsions. 